VEREINIGT MIT FOLIA HAEMATOLOGICA, NEUE FOLGE ZEITSCHRIFT FÜR DIE GESAMTE BLUTFORSCHUNG ORGAN DER DEUTSCHEN GESELLSCHAFT FÜR HÄMATOLOGIE ORGAN DER DEUTSCHEN GESELLSCHAFT FÜR BLUTTRANSFUSION

\title{
LEITARTIKEL
}

Aus dem Institut für Hämatologie, Abteilung Immunologie, GSF München (Leiter: PD Dr. S. Thierfelder)

\section{MLC-Eigenschaften, ein eigenes Histokompatibilitätssystem}

\author{
Von Wolfgang Mempel und Hans Grosse-Wilde*
}

Mischt man Lymphozyten zweier fremder Personen im Reagenzglas unter Zellkulturbedingungen, so erkennen sich die Zellen auf Grund genetisch determinierter Unterschiede als fremd und beginnen als Antwort zu proliferieren. Bei der Zellteilung wird neue DNS synthetisiert. Mit Hilfe von radioaktiv markierten DNSBausteinen (z. B. ${ }^{3} \mathrm{H}$-Thymidin) kann man die Proliferationsaktivität der Zellen messen. Die ersten Beobachtungen von Scbreck und Donnelly [23] griffen Bain [9] sowie Bach und Hirschborn [6] wieder auf und bauten die gemischte Lymphozytenkultur (Mixed Lymphocyte Culture - MLC) zu einem Gewebeverträglichkeitstest aus. Sie fanden, daß innerhalb von Familien die Zellen von eineiigen Zwillingen in der Kultur sich nicht gegenseitig anregten, während zweieiige Zwillinge und normale Geschwister in 1/4 der Fälle eine sehr starke Anregung, in der Hälfte der Fälle eine mittelstarke zeigten und nur in $1 / 4$ der Fälle keine Reaktion zu beobachten war [4,9]. Die Zellen nichtverwandter Personen zeigten im allgemeinen eine starke Reaktion.

Zur gleichen Zeit wurde der serologische Nachweis von Leukozytenantigenen des HL-A-Systems entwickelt [1]. Es zeigte sich, daß in Familien die Reaktionsweisen in der MLC mit den serologischen Antigenmustern übereinstimmten. Den Mendelschen Gesetzen folgend hatten in $25 \%$ zwei Kinder von den Eltern die gleichen HLA-Antigene geerbt und zeigten in der MLC keine Reaktion, in 50\% unterschieden sie sich auf Grund eines Haplotypes mit einer mittelstarken Reaktion in der MLC und in $25 \%$ auf Grund zweier Haplotypen mit starker Reaktion in der MLC [4]. Man nahm daher an, daß es die HL-A-Antigene selbst waren, die nicht nur zur Antikörperbildung Anlaß gaben, sondern auch direkt den Anstoß zur Proliferation in der MLC gaben. Die zeitraubendere Methode der MLC (Ergebnisse nach 4 Tagen) trat deshalb in der Gewebeverträglichkeitstestung gegenüber der einfacheren und weitaus schnelleren serologischen HL-A-Bestimmung (Ergebnisse nach $4 \mathrm{~h}$ ) in den Hintergrund. Lediglich in der Auswahl von Organspendern innerhalb einer Familie wurde

\footnotetext{
* Mitglieder des SFB 37.
}

Eingegangen am 7. 8. 1973. 
sie als zusätzlicher Test eingesetzt. $\mathrm{Da}$ es sich hierbei um Lebendspender handelte, konnte man die 4 bis 5 Tage, die die MLC benötigte, abwarten.

Bald jedoch traten Differenzen zwischen den Ergebnissen beider Methoden auf: In einigen Fällen zeigte es sich, daß Geschwister, obgleich sie serologisch identisch waren - also gleiche HL-A-Antigenmuster besaßen - in der MLC gegeneinander reagierten $[5,7,11,16,20,25]$. Als Beispiel sei die Familie Le. erwähnt, die wir eingehend untersuchen konnten [16] (Abb. 1a). Alle 5 Schwestern hatten vom Vater den gleichen Haplotyp geerbt, 4 Schwestern auch von der Mutter den gleichen Haplotyp, d. h. 4 waren serologisch identisch. Die fünfte Schwester unterschied sich von den übrigen in einem mütterlichen Haplotyp. Drei der identischen Schwestern

FAMILIE LE.

\begin{tabular}{|c|c|c|c|c|c|c|c|c|}
\hline$A$ & & HLA & $\mathrm{Cim}$ & $\mathrm{C}_{2} \mathrm{~m}$ & $\mathrm{C}_{3 m}$ & $\mathrm{C}_{4 \mathrm{~m}}$ & $\mathrm{Csm}$ & \multirow[b]{2}{*}{$q$} \\
\hline$\sigma^{\prime}$ & $\mathrm{Cl}_{1}$ & $\begin{array}{l}59-55 \\
11-50\end{array}$ & - & $\varnothing$ & n.d. & + & n.d. & \\
\hline \begin{tabular}{|l|l|}
59 & 55 \\
\end{tabular} & $\overrightarrow{C_{2}}$ & $\begin{array}{l}59-55 \\
11-50 \\
\end{array}$ & $\varnothing$ & - & $\varnothing$ & + & + & 3 \\
\hline \begin{tabular}{|l|l|}
3 & 68 \\
\end{tabular} & $\overline{C_{3}}$ & $\begin{array}{l}59-55 \\
11-50\end{array}$ & n.d. & $\varnothing$ & - & + & + & 11 \\
\hline Vater & $\mathrm{C}_{4}$ & $\begin{array}{l}59-55 \\
11-50\end{array}$ & + & + & + & - & $\varnothing$ & tter \\
\hline & $\mathrm{C}_{5}$ & $\begin{array}{r}59-55 \\
3-50\end{array}$ & n.d. & + & + & $\varnothing$ & - & \\
\hline
\end{tabular}

Abb. 1 a: Verhalten der Familie Le. in der MLC. MLC-Reaktionsmuster.

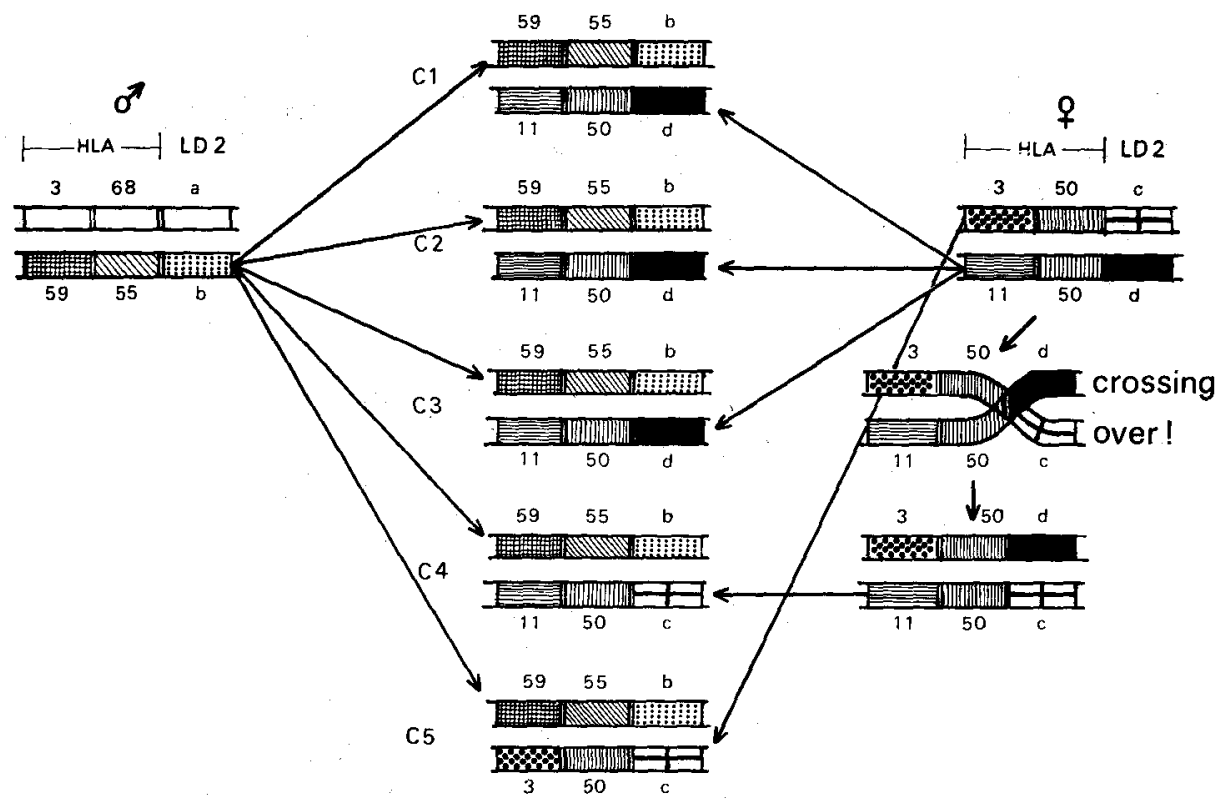

Abb. $1 \mathrm{~b}$ : Schematische Darstellung des crossing over.

Nach dem letzten Histocompatibility Workshop haben sich die Bezeichnungen für LD folgendermaßen geändert: Te $59 \rightarrow$ W $32 ;$ Te $55 \rightarrow$ W $15 ;$ Te $68 \rightarrow$ W $10 ;$ Te $50 \rightarrow$ W 5 . 
zeigten in der MLC keine Reaktion gegeneinander; die vierte Schwester dagegen reagierte sehr deutlich mit den drei übrigen und diese mit ihr. Diese vierte Schwester zeigte wiederum keine Reaktion mit der fünften Schwester und umgekehrt, obwohl sie serologisch different waren. Gegen die ersten drei Schwestern dagegen reagierte die fünfte Schwester ganz normal.

Die beste Erklärung für das Verhalten in dieser Familie war die Annahme 1. getrennter Genorte auf dem gleichen Chromosom, einmal für die serologisch definierten HL-A-Antigene, zum anderen für das Verhalten in der MLC und 2. ein crossing over zwischen diesen beiden Eigenschaften bei dem mütterlichen Chromosom der vierten Schwester (Abb. 1b). Die vierte Schwester hatte somit die gleichen serologisch definierten HL-A-Antigene wie die ersten drei Schwestern von ihrer Mutter geerbt, dagegen eine MLC-Eigenschaft, die mit der fünften Schwester übereinstimmt. Es ergibt sich danach zwischen den Schwestern eine unterschiedliche Kompatibilität, gemessen entweder an serologischen Kriterien oder am MLCVerhalten.

Die Chromosomenregion des Menschen, auf der die wichtigsten Transplantationssysteme vereinigt sind, beginnt sich etwas weiter aufzuhellen. Allerdings sind wir von einer genauen Kenntnis noch weit entfernt. Wir sehen nur einige Spitzen im Nebel-
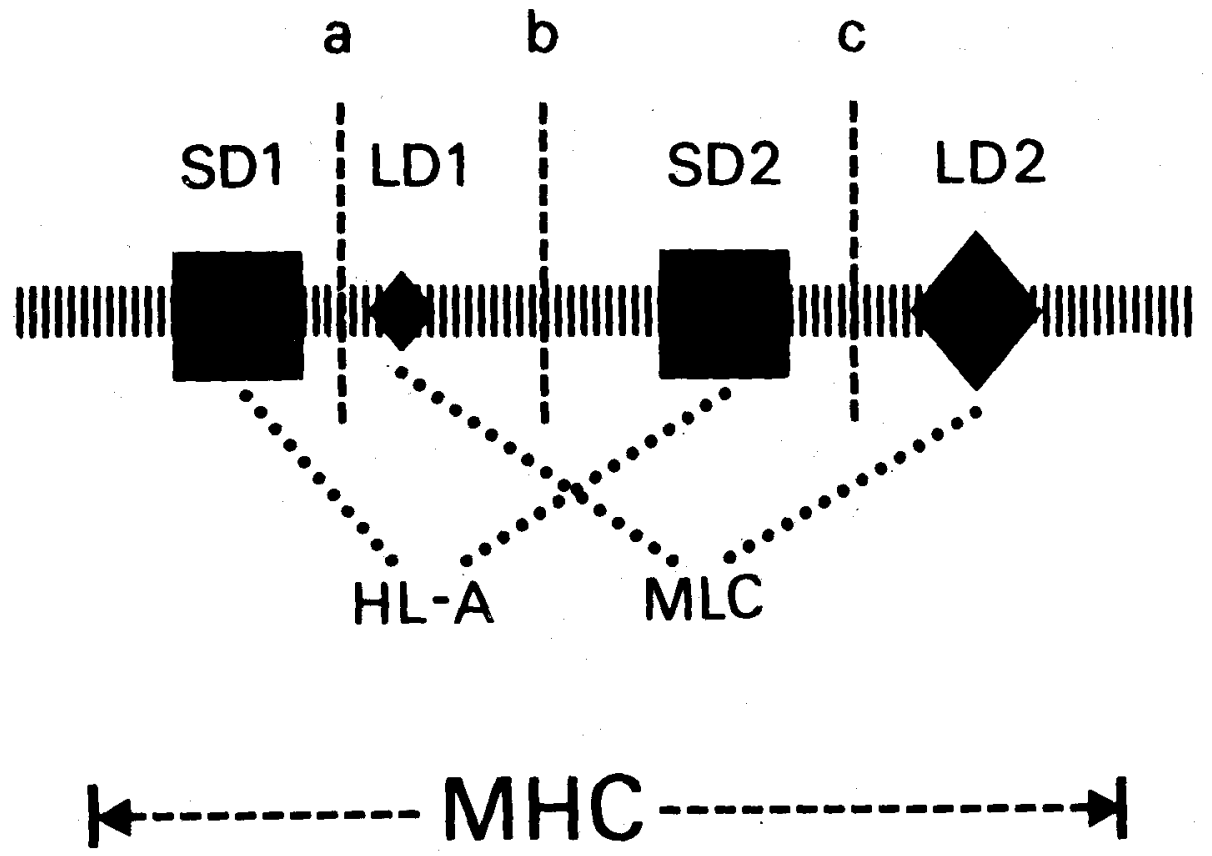

a. b, $c$ : bisher beobachtete Crossing-over

$\mathrm{SD}=$ serological.defined

$\mathrm{LD}=$ lymphocyte defined

$\mathrm{MHC}=$ major histocompatibility complex

Abb. 2: Chromosomenregion für die wichtigsten Transplantationssysteme des Menschen. 
meer, wissen aber noch nichts oder nur sehr wenig über die Beziehung der einzelnen Punkte zueinander [8], obwohl die Chromosomenregion in ihrer Gesamtheit für die Transplantatabstoßung offensichtlich eine entscheidende Rolle spielt. Ceppellini [10] nannte sie deshalb „Transplantation-Super-Gene“, F. H. Bacb [8] prägte dafür den Namen „MHC“ [Major Histocompatibility Complex]. Beide Ausdrücke lassen Raum für weitere genetische Systeme, die im Zusammenhang mit Transplantaterkennung und -abstoßung stehen. Bisher können wir nur zwei Systeme festlegen (Abb. 2). Das erste besteht aus zwei loci des HL-A-Systems. Die Antigene dieses Systems sind, da sie serologisch faßbar sind, bisher besser exforscht. (Die Genorte werden einem Vorschlag von F. Bach zufolge SD1 = serological defined I. HL-A locus und $\mathrm{SD} 2=\mathrm{II}$. HL-A locus bezeichnet). Der Abstand der beiden Genorte ist mit einer crossing-over-Häufigkeit von 0,85\% nicht sehr groß. Der stärkste MLC locus ist nach ersten sehr ungenauen Schätzungen vom II. HL-A locus (SD2) gleichweit entfernt wie dieser vom I. HL-A locus. $\mathrm{Ob}$ es sich um einen einzelnen definierten Genort oder um mehrere engbenachbarte Genorte handelt, ist im Augenblick nicht sicher zu entscheiden. Daten aus Mäuseversuchen lassen die Interpretation einer Genanhäufung zu [14]. Zwischen beiden HL-A loci, in Nachbarschaft zum ersten HL-A locus, liegt wahrscheinlich ein zweiter, schwächerer MLC locus [11, 17,18,21,24]. Die MLC loci werden als LD, lymphocyte defined, bezeichnet, da sie durch Lymphozytenreaktion definiert sind. LD2 liegt in der Nähe von SD2, LD1 in der Nähe von SD1 (s. Abb. 2). Die meisten bisherigen Befunde lassen sich in dieses System einordnen. In der Familie hat man gefunden, daß bei einem crossing over zwischen I. und II. HL-A locus im allgemeinen Differenzen am II. HL-A locus zu einer Stimulation in der MLC führen. Unterschiede für Antigene des I. HL-A locus (SD1 + LD1) führen nur in einem Teil der Fälle zu einer schwachen Stimulation. Erfolgt das crossing over zwischen II. HL-A locus und starkem MLC locus, ergibt sich eine Stimulation zwischen HL-A-serologisch identischen Geschwistern einerseits und eine Nichtstimulation zwischen Geschwistern mit einem HL-A-Haplotyp Unterschied.

In nichtverwandten Kombinationen ist durch die vielen crossing-cover-Möglichkeiten im Verlauf der Generationsfolge von vornherein zu erwarten, daß eine bestimmte HL-A-Kombination mit verschiedenen MLC-Eigenschaften gekoppelt ist. Es ist deshalb nicht verwunderlich, daß 90\% aller HL-A-identischer unverwandter Kombinationen in der MLC eine Stimulation zeigen. Allerdings reagieren 10\% nicht gegeneinander, d. h. sie tragen neben identischen, serologisch erfaßbaren HL-AAntigenen auch identische Eigenschaften des MLC-Systems (SD und LD identisch). Da eine Nichtstimulation zwischen willkürlich gewählten Partnern außerordentlich selten vorkommt, weist diese $10 \%$-MLC-Identität bei HL-A-Identität auf eine gewisse bevorzugte Koppelung zwischen HL-A-Antigenen und MLC-Eigenschaften hin. Einen weiteren Hinweis ergab der Vergleich von mittleren Stimulationshöhen zwischen Personen, die sich in einem, zwei oder mehr HL-A-Antigenen unterschieden. Mit steigender antigener Differenz ergibt sich auch ein Anstieg der mittleren Stimulationstate, was - wenn man davon ausgeht, daß es nicht die HL-A-Antigene selbst sind, die eine Stimulation bewirken - für eine gewisse Koppelung von HL-ASystem und MLC-System spricht. Dabei machen Differenzen am II. HL-A-locus allein den Großteil der Stimulationssteigerung aus [17,18], d. h. die MLC-Eigen- 
schaft ist in erster Linie mit dem II. HL-A locus gekoppelt. Differenzen am I. locus machen nur einen geringen, aber dennoch erkennbaren Anstieg. Dies unterstützt die Annahme, daß in der Nähe des I. locus ein zweiter schwächerer MLC-locus(LD1) liegt.

LD1 ist also eine schwache, LD2 eine starke MLC-Region. Aus neueren Statistiken der Nierentransplantationen in Eurotransplant [22] und in England [19] geht interessanterweise hervor, daß auch die Úberlebenszeit der Nierentransplantate mit der Übereinstimmung der Antigene am II. HL-A locus korreliert, unabhängig von Differenzen am I. HL-A locus. Experimentelle Hauttransplantate zwischen nichtverwandten Personen [22], die in allen 4 HL-A-Antigenen übereinstimmten, zeigten eine längere Überlebenszeit, wenn die Zellen der Partner sich in der MLC nicht oder nur wenig stimulierten, als diejenigen, deren MLC hohe Werte aufwiesen. Beides deutet darauf hin, daß den MLC-Eigenschaften oder Eigenschaften, deten Gene in enger Nachbarschaft zu den MLC-Genen liegen, bei der Transplantatabstoßung eine große Bedeutung zukommt. Man wird also in Zukunft die Ergebnisse der MLC stärker als bisher in der Spenderauswahl berücksichtigen müssen. Leider ist das größte Hindernis zu einer Routineanwendung der große Zeitaufwand. Da man Nieren heute schon ohne Schaden $72 \mathrm{~h}$ konservieren kann, ergibt sich vielleicht die Möglichkeit, doch noch eine MLC durchzuführen. Die Suche nach Kurzzeitmethoden ist bisher erfolglos geblieben. Weiterhin versucht man das Problem serologisch zu lösen. Allerdings ist bisher noch nicht völlig klar, ob die Genprodukte der MLCloci Antigene im klassischen Sinn darstellen, d. h. ob sie zu einer Antikörperbildung Anlaß geben können. Einige Tierversuche sprechen dafür und auch beim Menschen gibt es einige Hinweise [12].

Solange es Seren gegen die MLC-Eigenschaften nicht in genügender Reinheit und Menge gibt, muß man versuchen, das System mit Hilfe der umständlicheren KulturMethode aufzuklären. Besonders interessant erscheint die Aufklärung des Polymorphismus des Systems und die Frage der bevorzugten Koppelung mit dem HL-ASystem. Denn 10\% der HL-A-identischen nichtverwandten Personenpaare stimulieren sich nicht. Testet man größere Gruppen HL-A-identischer Personen, so findet sich innerhalb jeder bisher untersuchten Gruppe eine kleinere Gruppe von Personen, die sich alle untereinander nicht stimulieren. Bei den häufigsten Phänotypen HL-A $1-3-7-8$ und 1-2-7-8 waren dies 5 aus 19, bzw. 5 aus 14 [18].

Gehört jemand der bevorzugten MLC-negativen Gruppe eines HL-A-Phänotypes an, so hat er in ca. $30 \%$ die Chance, daß ein Partner, wenn er schon zufällig HL-A-identisch ist, auch noch zusätzlich MLC-identisch ist. Außerhalb der bevorzugten Gruppe ist die Chance sehr viel geringer, wenn auch nicht Null. Denn gelegentlich findet man außerhalb der MLC-negativen Gruppe auch noch Einzelpaare, die sich gegenseitig nicht stimulieren. Man kann also auch jetzt schon bei Transplantatempfängern, sofern man für den entsprechenden HL-A-Typ auch den bevorzugten MLC-Typ kennt, etwas über die Chance aussagen, ein annähernd ideales Organ zu erhalten. Allerdings sind HL-A-identische Transplantate an sich schon extrem selten. Aber vielleicht ist HL-A-Identität nicht in jedem Fall erforderlich. Es konnte z. B. gezeigt werden, daß die MLC-negative Gruppe des HL-A-Types 1-3-7-8 mit der des HL-A-Types 1-2-7-8 gegenseitig keine Reaktion hervorrief, d. h. beide Gruppen haben die gleichen MLC-Eigenschaften, obwohl sie eine HL-ADifferenz aufweisen. 
Um diese MLC-Eigenschaften besser festlegen zu können, erfolgt z. Zt. eine Suche nach Personen, die homozygot für eine bestimmte MLC-Eigenschaft sind: Man findet hin und wieder Familien, in denen beide Eltern einen HL-A-Haplotyp gemeinsam haben. Es können dann Kinder auftreten, die entweder mit einem der Eltern HL-Aidentisch sind oder aber solche, die homozygot für diesen Haplotyp sind. Eine kritische Kombination ist nun die HL-A-identische Eltern-Kind-Situation. Ist diese Kombination MLC-negativ, so ist anzunehmen, daß die MLC-Eigenschaften, die an die den Eltern gemeinsamen HL-A-Haplotypen gekoppelt sind, auch die gleichen sind. Das Kind muß ja den fraglichen Haplotyp vom anderen Elternteil geerbt haben. Die Zellen der homozygoten Kinder müßten dann theoretisch Einwegreaktionen gegen die Eltern und gegen heterozygote Geschwister zeigen, d. h. sie sollten sich durch die heterozygoten Partner anregen lassen, ihrerseits aber diese nicht anregen.

In vielen untersuchten Fällen kommt es nun zu dieser erwarteten Einwegreaktion, aber leider nicht in allen. Die Gründe, warum auch die homozygoten Zellen in einigen Fällen in der Lage sind, ihre heterozygoten Partner zu stimulieren, sind noch nicht ganz klar. Es werden verschiedene Erklärungen gegeben, von denen keine völlig befriedigend ist.

Trotzdem läßt sich aber mit solchen Zellen arbeiten und in einer ersten Studie mit homozygoten Zellen, die den HL-A-Typ 3-7 tragen und deren MLC-Eigenschaft „Pi“ genannt wurde, konnte gefunden werden, daß in einer Personengruppe, die nach dem HL-A-Antigen 7 ausgewählt war, die MLC-Eigenschaft „Pi“ in ca. 36\% auftritt, während sie in einer unausgewählten Kontrollgruppe in ca. 14\% auftritt. Es ergibt sich also eine Verschiebung des Koppelungsgleichgewichtes zugunsten des HL-A-7-Types. Eine Störung des Koppelungsgleichgewichts ist uns von den HL-AAntigenen selbst auch schon bekannt und erste vorsichtige Berechnungen zeigen, daß die Koppelung zwischen HL-A 7 und MLC-Typ „,Pi“ etwa die gleiche ist, wie zwischen HL-A 3 und HL-A 7. Dies sind jedoch erste Anfänge und viel bleibt noch zu tun. Eine sehr wichtige Aufgabe der Zukunft wird sein festzustellen, ob gewisse Krankheiten, die eine deutliche Korrelation zum HL-A-System zeigen [3], auch mit bestimmten MLC-Eigenschaften gekoppelt sind. Erste Untersuchungen an MS-Patienten [13] und an M. Bechterew-Patienten (eigene Beobachtung) sprechen dafür.

Literatur: 1 Albert, E. D.: Blut 1973. - 2 Albert, E. D., W. Mempel and H. Grosse-Wilde: Transpl. Proc. 1973, im Druck. - 3 Albert, E. D., K. Harms, R. Wank, I. SteinbauerRosenthal and S. Scholz: Transpl. Proc. 1973, im Druck. - 4 Albertini, R. J. and F. H. Bach: J. Exp. Med. 128, 639 (1968), - 5 Amos, D. B. and F. H. Bach: J. Exp. Med. 128, 623 (1968). - 6 Bach, F. H. and K. Hirschhorn: Science 149, 813 (1964). - 7 Bach, F. H., R. J. Albertini, D. B. Amos, R. Ceppelini, P. L. Martinez and V. C. Miggiano: Transpl. Proc. 1, 339 (1969). - 8 Bach, F. H.: Transpl. Proc. Vol. No.1, 23 (1973). - 9 Bain, B. and L. Lowenstein: Science 145, 1315 (1964). - 10 Ceppellini, R.: Progr. in Immunology I, 973, Acad. Press 1972. - 11 Eijsvoogel, V. P., J. J. van Rood, E. D. du Toit and P. Th. A. Schellekens: Eur. J. Immunol. 2, 413 (1972). - 12 Götze, D., R. A. Reisfeld and J. Klein: J. Exp. Med. 1973, im Druck. - 13 Jersild, C., B. Dupont, T. Fog, G. S. Hansen, L. S. Nielsen, A. Svejgaard and Mithomsen: Transpl. Proc. 1973, im Druck. - 14 Klein, J. and J. M. Park: J. exp. Med. 137, 1213 (1973). - 15 Mempel, W.: Klin. Wschr. 50, 287 
(1972). - 16 Mempel, W., E. D. Albert, and A. Burger: Tissue Antigens 2, 250 (1972). 17 Mempel, W., H. Grosse-Wilde, E. D. Albert and S. Thierfelder: Transpl. Proc. Vol. V, No. 1, 401 (1973). - 18 Mempel, W., H. Grosse-Wilde, P. Baumann, B. Netzel, I. Steinbauer-Rosenthal, S. Scholz, J. Bertrams and E. D. Albert: Transpl. Proc. 1973, im Druck. 19 Oliver, R. T. D., J. A. Sachs and H. Festenstein: Transpl. Proc. V, No. 1, 245 (1973). 20 Plate, J. M., F. E. Ward and D. B. Amos: Histocompatibility Testing 531 Munksgaard Copenhagen 1970. - 21 Revillard, J. P.: Transpl. Proc. 1973, im Druck. - 22 van Rood, J. J., C. T. Koch, J. P. van Haaf, A. van Leeuwen, J. G. van der Tweel, E. Frederiks, H. M. A. Schippers, G. Hendriks and G. J. van der Steen: Transpl. Proc. V, No. 1, 409 (1973). - 23 Schreck, R. and W. J. Donnelly: Blood 18, 561 (1961). - 24 Thorsby, E.: Transpl. Proc. 1973, im Druck. - 25 Yunis, E. J., G. M. Plate, F. I. Ward, H. F. Seigler and D. B. Amos: Transpl. Proc. Vol. 3, 1, 118 (1971).

Anschr. d. Verff.: Dr. W. Mempel und Dr. H. Grosse-Wilde, 8 München 2, Landwehrstraße 61. 